\title{
Prospects for the Primakoff measurement of the pion polarizability at COMPASS
}

\author{
Jan Friedrich ${ }^{* \dagger}$ \\ Uni Erlangen / TU München \\ E-mail: jan.friedrich@ph.tum.de \\ for the COMPASS collaboration
}

Using high rate capability in conjunction with high precision, COMPASS can significantly contribute to the physics studied at very low momentum transfers as in Primakoff reactions, that is, the scattering of hadrons off the quasi-real photons forming the Coulomb field of nuclei. Most importantly, it has the unique chance to clarify the question of the pion polarisability, where an unprecedented level of precision is in reach.

6th International Workshop on Chiral Dynamics

July 6-10 2009

Bern, Switzerland

\footnotetext{
* Speaker.

$\dagger$ work supported by the DFG cluster of excellence "Origin and Structure of the Universe" (www.universe-cluster.de)
} 

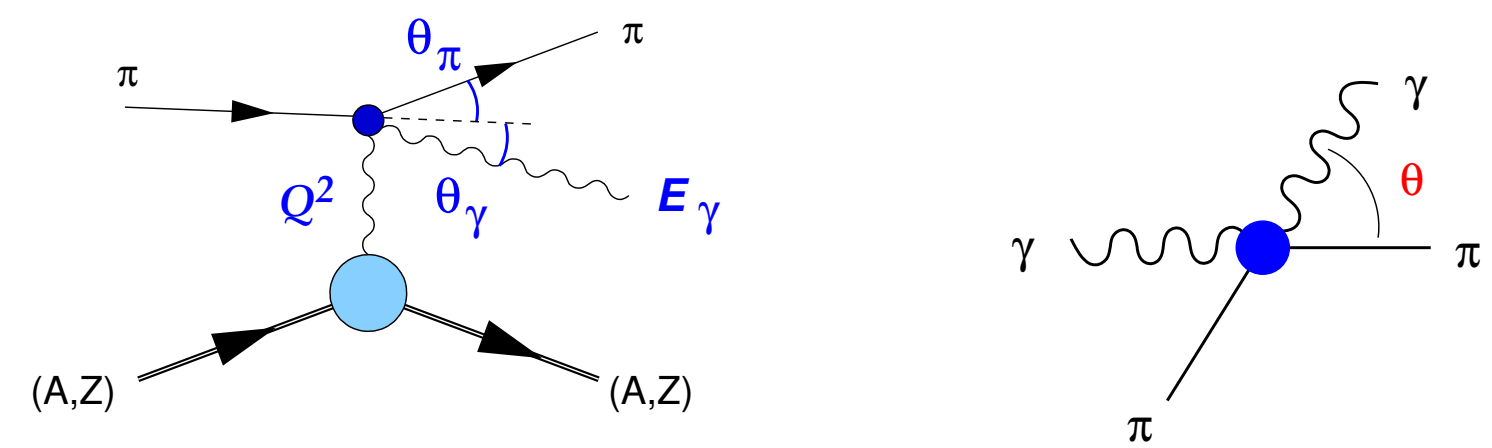

Figure 1: Pion Compton reaction (right graph) embedded in the Primakoff reaction (left graph) on a nucleus of charge $Z$. Kinematical quantities in the laboratory and the $\mathrm{CM}$ scattering angle are indicated.

\section{Pion polarisability}

The electric and magnetic dipole polarisabilities of the charged pion, $\alpha_{\pi}$ and $\beta_{\pi}$, describe the interaction of the pion with external electromagnetic fields in the low-field quasi-static limit. They are fundamental parameters of pion physics, and the comparison of theoretically predicted and directly measured values provides a stringent test for various theoretical approaches like dispersion or quantum chromodynamics (QCD) sum rules and quark confinement models. These theoretical predictions disagree with each other and lie in the range $(2-8) \times 10^{-4} \mathrm{fm}^{3}$ for the absolute values of $\alpha_{\pi}$ and $\beta_{\pi}[1,2,3]$.

In the systematic QCD low-energy expansion of chiral perturbation theory $(\chi \mathrm{PT})$, the pion is interpreted as the Goldstone boson of the underlying chiral symmetry, and its experimentally determined properties test this systematic approach. $\chi \mathrm{PT}$ has established a firm prediction of the pion polarisabilities, including higher-order effects and an error estimate (see [4, 5]), $\alpha_{\pi}-\beta_{\pi}=$ $(5.7 \pm 1.0) \times 10^{-4} \mathrm{fm}^{3}$. The sum of the pion polarisabilities is small, taking a non-zero value only starting from a 2-loop $\chi \mathrm{PT}$ calculation, $\alpha_{\pi}+\beta_{\pi}=0.16 \times 10^{-4} \mathrm{fm}^{3}$.

The actual dispute on the compatibility of $\chi \mathrm{PT}$ with the dispersion relation approach for the pion polarisability (see e.g. these proceedings $[6,7]$ ) demonstrates the urgent need for experimental clarification.

The method of radiative $\pi^{+}$photoproduction off the proton, most recently used at MAMI [8], has confirmed the earlier finding of the Serpukhov experiment [9], employing the Primakoff method (cf. Sec.2). In the MAMI data, as in the Serpukhov, the pion polarisability is found about twice as large as predicted by $\chi \mathrm{PT}$.

\section{Primakoff measurement of pion Compton scattering}

A high-energetic pion beam allows to study scattering reactions within the pion lifetime. Scattering off nuclei at small momentum transfer $Q^{2}$ to the nucleus, in the range $\leq 0.001 \mathrm{GeV}^{2} / c^{2}$ dominates the electromagnetic interaction, as the small momentum transfer corresponds to large impact parameters compared to the extension of the nucleus. So effectively, in this regime the 


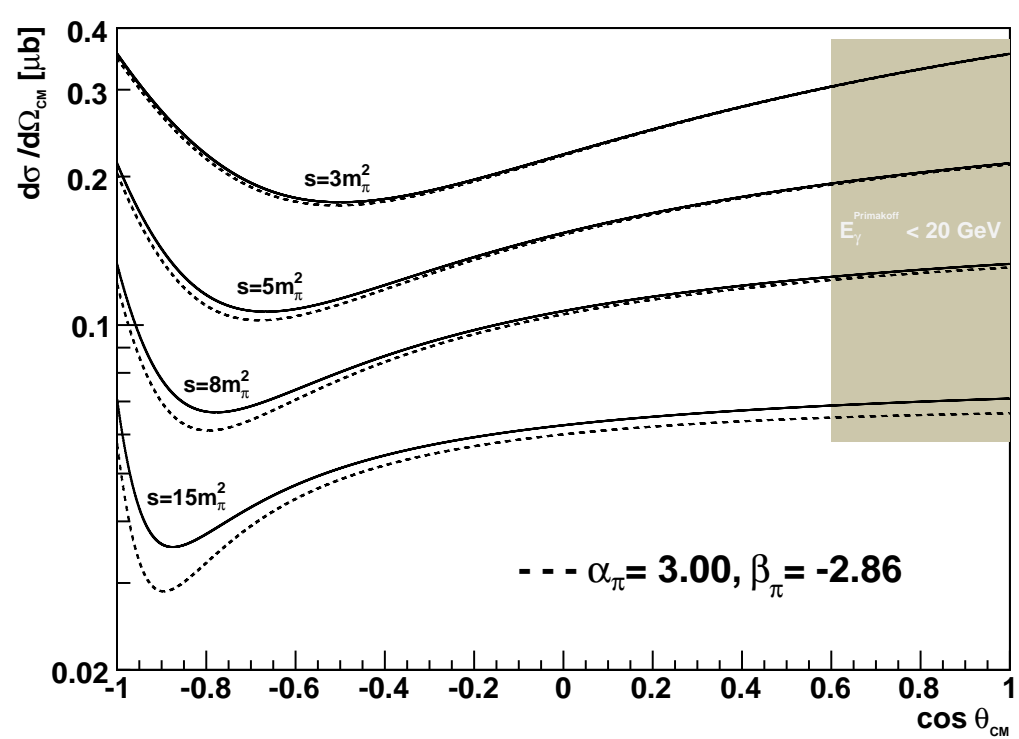

Figure 2: Polarisability effect in pion Compton scattering on the differential cross section, depending on the scattering angle $\theta_{\mathrm{CM}}$ in the $\pi \gamma$ centre-of-momentum system for different squared $\mathrm{CM}$ energies $s$. The continuous line shows the differential cross section in the pointlike case, and the dashed line indicates the prediction of $\chi \mathrm{PT}$ (other contributions, as those from chiral loops, are suppressed here).

pions scatter off photons of the Coulomb field of the nucleus, which is referred to as Primakoff reaction [10], and in the process

$$
\pi^{-} Z \rightarrow \pi^{-} Z \gamma
$$

the pion Compton scattering reaction is embedded, as depicted in Fig. 1. The cross section for this reaction, treating the nuclear vertex in the equivalent photon approximation, is given by

$$
\frac{d \sigma}{d s d t d Q^{2}}=\frac{\alpha}{\pi\left(s-m_{\pi}^{2}\right)} \cdot F_{Z}^{2}\left(Q^{2}\right) \cdot \frac{Q^{2}-Q_{\min }^{2}}{Q^{4}} \cdot \frac{d \sigma_{\pi \gamma}}{d t}
$$

where $Q_{\min }=\left(s-m_{\pi}^{2}\right) / 2 E_{\text {beam }}, s$ and $t$ are the Mandelstam variables in the $\pi \gamma$ system, and the pion polarisabilities enter via

$$
\frac{d \sigma_{\pi \gamma}}{d \Omega_{c m}}=\frac{\alpha^{2}\left(s^{2} z_{+}^{2}+m_{\pi}^{4} z_{-}^{2}\right)}{s\left(s z_{+}+m_{\pi}^{2} z_{-}\right)^{2}}-\frac{\alpha m_{\pi}^{3}\left(s-m_{\pi}^{2}\right)^{2}\left[z_{-}^{2}\left(\alpha_{\pi}-\beta_{\pi}\right)+\frac{s^{2}}{m_{\pi}^{4}} z_{+}^{2}\left(\alpha_{\pi}+\beta_{\pi}\right)\right]}{4 s^{2}\left(s z_{+}+m_{\pi}^{2} z_{-}\right)}
$$

with $z_{ \pm}=1 \pm \cos \theta$ and $\theta$ the scattering angle in the $\mathrm{CM}$ system of the outgoing $\pi \gamma$ pair.

The effect of the difference of electric and magnetic polarisability $\left(\alpha_{\pi}-\beta_{\pi}\right)$ is strongest under backward angles (for this reason, this quantity is also referred to as backward polarisability), while the forward polarisability $\left(\alpha_{\pi}+\beta_{\pi}\right)$ influences the cross section at large $\cos \theta$. While the latter forward polarisability is expected to be small values (cf. Sec. 1), it is enhanced in the cross section by the $s^{2} / m_{\pi}^{4}$ factor. Consequently, measuring the energy and angular dependence of the cross section allows an extraction of the two polarisabilities separately. 


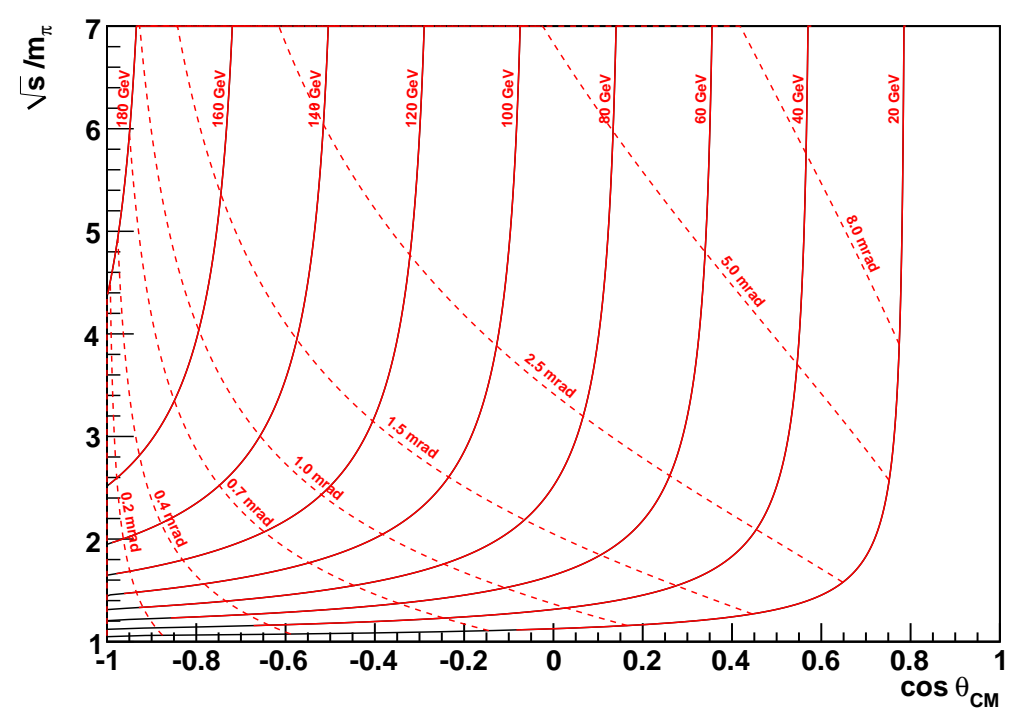

Figure 3: Kinematical relation of the Mandelstam variables $s$ and $t$, appearing in the cross section formulae, with the laboratory of the outgoing photon. For COMPASS measurements, the photon energy threshold will lie at about $40-60 \mathrm{GeV}$.

The polarisability effect as given by Eq. 2.3 is depicted in Fig. 2. For the case of COMPASS kinematics with a $190 \mathrm{GeV}$ incoming pion beam, Fig. 3 shows the kinematical relation with the laboratory quantities of the produced photon. The physically interesting region includes photons with energies in the range from 20 to $180 \mathrm{GeV}$ at angles from 0 to about $5 \mathrm{mrad}$ with respect to the incoming beam pion direction, referring to the physically interesting region $-1<\cos _{\mathrm{CM}}<0.6$ and $1.5<\sqrt{s}<4$.

\section{Primakoff measurements at COMPASS}

The COMPASS experiment at CERN is a 2-stage magnetic spectrometer equipped with modern detector and data acquisition technology for large-acceptance measurements with high intensity beams and high interaction rates. The setup is described in [11]. For measuring Primakoff reactions as process 2.1, two features of COMPASS play a key role: Firstly, the excellent tracking capability for the incoming and outgoing pions, allowing the determination of the scattering angle with a precision better than $50 \mu \mathrm{rad}$, and secondly the electromagnetic calorimetry for energetic and spatial reconstruction of the produced photons. Furthermore, the elaborated muon identification system permits an identification of muon-induced reactions similar to 2.1. The possibility to switch to muon beam can be used in order to study systematic effects, since the cross section for the analogous process for the point-like muons is fully determined by QED.

The study of Primakoff reactions in COMPASS was first started in a pilot hadron run in 2004 using $190 \mathrm{GeV} \pi^{-}$and $\mu^{-}$beams impinging on a solid $\mathrm{Pb}$ target, segmented in longitudinal direction. Additional samples for $\mathrm{Cu}$ and $\mathrm{C}$ targets were also collected. The total amount of pion Primakoff events from the lead target was about 60,000 events for photon energies above $40 \mathrm{GeV}$. 


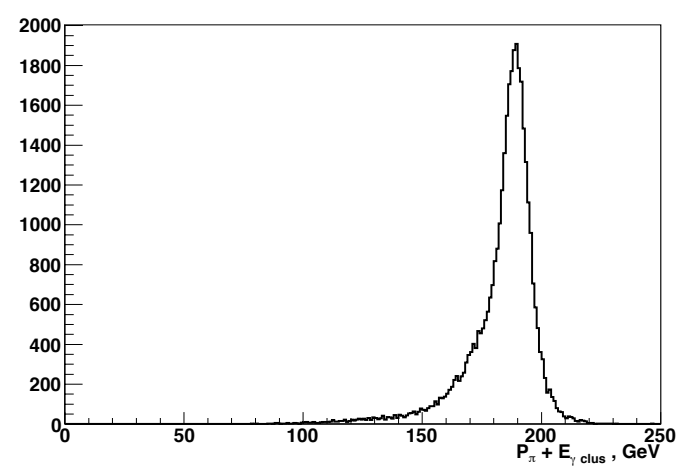

Figure 4: Exclusivity peak of $E_{\gamma}+E_{\pi}$ for $\pi \gamma$ events.

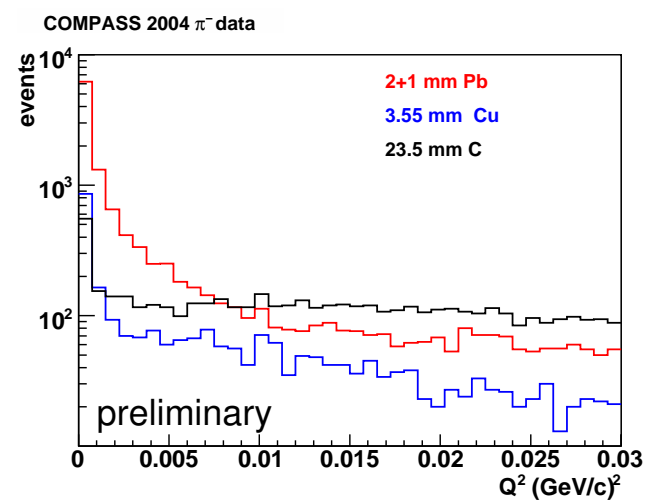

Figure 5: $Q^{2}$-distribution for different targets. Primakoff events clearly dominate near $Q^{2}=0$.

Instabilities in the amplification of many cells of the electromagnetic calorimeter connected with saturation effects, and some inefficiency in the trigger component for the scattered pions, turned out to cause significant systematic uncertainties of the measurement and did not allow us to extract a reliable value for the pion polarisabilities from the 2004 data. However, the measurement allowed to address the full experimental procedure and established the feasibility of a much improved measurement.

The most important achievements of the present analysis are, on the one hand, the selection of Primakoff reactions with the selection of exclusive $\pi \gamma$ events (Fig. 4) and the identification of the Primakoff peak in the $Q^{2}$-distribution (Fig. 5). Normalizing to the luminosities realized with the different targets, we found the strength of the Primakoff peak to follow the theoretically expected $Z^{2}$-dependence. On the other hand, we determined the important parameters of our setup such as resolutions and Monte Carlo control of detector efficiencies and identified the main sources of background, both, non-separable physics processes (the ratio of Primakoff signal to diffractive background for different targets as presented in Fig.5), and backgrounds stemming from beam impurities for which a set of procedures for background reduction has been developed.

Corrections to the pure tree-level Primakoff cross section come from the Compton vertex, multiple photon exchange, vacuum polarization and nuclear charge screening by atomic electrons and have also been calculated. It was found that the multiple photon exchange introduces a large correction (see $[12,13]$ ) for the lead target, estimated to be about 5\%, making it favorable to measure on lighter nuclei like Nickel where this correction lies below 1\%. The transition region to higher $Q^{2}$ was investigated within the Glauber model in the work of [14, 15], in which the influence of the strong interaction on the $Q^{2}$ spectrum is addressed.

\section{Prospects for new measurements}

In November 2009, a second data taking period for Primakoff measurements is ongoing. The readout electronics of the electromagnetic calorimeter has been refurbished, and was used in the 2009 data taking of diffractive reactions aiming at meson spectroscopy, where it has proven a much 
better performance as compared to the 2004 data as presented in Sec. 3. An additionally installed laser system permits the control of the amplifications of the individual cells during the beam time.

A new component is the digital calorimeter trigger, it delivers a trigger signal as a function of the energy sum of the relevant cells of the calorimeter, which makes it an ideal tool for the intended Primakoff measurement.

The planned luminosity exceeds the statistics of the previous COMPASS data taken in 2004, which turned out to be comparable to the Sepukhov statistics, by about a factor of 10 . This will allow the determination of the polarisability difference $\alpha_{\pi}-\beta_{\pi}$ to a precision comparable to the theoretical uncertainty as outlined in Sec. 1, assuming a negligible contribution from the sum of polarisabilities and so, introducing the constraint $\alpha_{\pi}+\beta_{\pi}=0$ in the analysis. This, effectively, means that it is sufficient to observe the deviation of the cross section from the pointlike expectation with increasing energy of the produced photon only, integrating over the angular dependence.

It will be more ambitious to disentangle the magnetic and electric contribution separately, since the cross section behaviour must then be studied in both its dependence on the energy and angles of the outgoing particles, as explained in Sec. 2.

In order to reach a comparable statistical error on the two polarisabilities independently, a beam time of about 4 weeks at COMPASS is to be envisaged. During such a new beam time, it will be possible to obtain also first data on Compton scattering of the charged kaon, using also the kaon component of the incoming beam. In analogy to the described method for the pion, the kaon polarisability could be determined for the first time.

\section{References}

[1] J. Portoles, M.R. Pennington, The second Daథne Physics Handbook 579 (1999), hep-ph/9407295.

[2] C.A. Wilmot, R.H. Lemmer, Phys. Rev. C65, 035206 (2002).

[3] L.V. Fil'kov, V.L. Kashevarov, Phys. Rev. C73, 035210 (2006).

[4] N. Kaiser, J. M. Friedrich, Eur. Phys. J. A36, 181 (2008).

[5] J. Gasser, M. A. Ivanov, M. E. Sainio, Nucl. Phys. B745, 84 (2006).

[6] B. Pasquini, D. Drechsel, S. Scherer, these proceedings, hep-ph/0908.4260

[7] L. Fil'kov, V. Kashevarov, these proceedings, hep-ph/0909.4849v2

[8] J. Ahrens et al., Eur. Phys. J. A23, 113 (2005).

[9] Y. Antipov et al., Phys. Lett. B121, 445 (1983).

[10] H. Primakoff, Phys. Rev. 81, 899 (1951).

[11] The COMPASS experiment at CERN, Nucl. Instrum. Meth. A577, 455 (2007).

[12] N. Kaiser, J. M. Friedrich, Nucl. Phys. A812, 186 (2008).

[13] N. Kaiser, J. M. Friedrich, Eur. Phys. J. A39, 71 (2009).

[14] G. Fäldt, U. Tengblad, Phys. Rev. C79, 014607 (2009).

[15] T. Walcher Prog. Part. Nucl. Phys. 61, 106 (2008). 